\title{
Lymphatic obstruction in rheumatoid arthritis: a cause for upper limb oedema
}

\author{
R. T. D. DE SILVA, D. M. GRENNAN, AND D. G. PALMER \\ From the Departments of Radiology and Medicine, University of Otago Medical School, Dunedin, New Zealand
}

SUMMARY Peripheral lymphatic obstruction has been demonstrated by lymphangiography as a cause of upper limb oedema in 2 patients with seronegative rheumatoid disease.

A small proportion of patients with rheumatoid arthritis develop peripheral oedema which appears to be more widespread than can be attributed to inflammatory synovitis involving the regional joints and which cannot be related to general factors such as anaemia, hypoalbuminaemia, or generalised fluid retention. The results of previous studies have suggested that such oedema may be the result of venous obstruction (Swinburne, 1964), of a generalised increase in capillary permeability (Jayson and Barks, 1971), or of lymphatic obstruction (Kalliomaki and Vastamaki, 1968). Some of these previous studies have examined patients with oedema of the lower limbs only (Swinburne, 1964; Jayson and Barks, 1971; Jayson et al., 1971), a situation in which such factors as immobility and generalised fluid retention are difficult to exclude.

In contrast, when oedema of the hands and forearms accompanies rheumatoid arthritis, such causes of fluid retention can ordinarily be discounted if dependent oedema does not involve the lower limbs. In this paper 5 rheumatoid patients with oedema of the upper limbs and 2 with oedema of the lower limbs were investigated by lymphangiography. In 2 of the patients with hand and forearm oedema unequivocal lymphangiographic evidence of lymphatic obstruction was found.

\section{Patients and methods}

PATIENTS WITH LYMPHANGIOGRAPHIC EVIDENCE OF LYMPHATIC OBSTRUCTION Case 1 was a 56-year-old woman who presented with an 8-month history of shoulder pain and a 6-week history of pain in the knees. Bilateral knee effusions

Accepted for publication 24 April 1979.

Correspondence to Associate Professor D. G. Palmer, Wellcome Medical Research Institute, Department of Medicine, University of Otago Medical School, PO Box 913, Dunedin, New Zealand. were present. The sheep cell agglutination test was consistently negative, but articular erosions appeared. Swelling over the dorsum of the right wrist was noted 9 months later. Six years after the onset of symptoms the right forearm and hand became diffusely swollen with pitting of the oedema, and some 4 months later the left forearm and hand were similarly involved. Finger movements remained full despite erosive changes having developed in the wrists and various small joints of the hands. The swelling was said to fluctuate but on one occasion was observed to extend to the elbows. On another occasion, after aspiration of a knee joint and injection of a corticosteroid preparation the upper arm oedema was observed to resolve within 48 hours of the procedure only to reappear gradually over the subsequent weeks. The patient was receiving sodium aurothiomalate and soluble aspirin at the time forearm oedema appeared.

Case 2 was a 57-year-old woman who developed seronegative erosive rheumatoid arthritis 12 years before presenting with diffuse pitting oedema of the right hand and forearm. Her treatment at this time consisted of sodium aurothiomalate and ibuprofen. The course of her arthritis had tended to be episodic, and multiple joints including the shoulders, elbows, wrists, knees, and ankles had been involved. Involvement of the flexor sheaths of the fingers and the extensor sheaths at the wrists had been noted.

Swelling of the right forearm persisted for 6 months before resolving completely, but this was shortly followed by similar swelling of the left hand and forearm. Nine months later both forearms were noted to be simultaneously involved, and after yet a further 3 months all swelling had completely disappeared. The episodes of forearm oedema were not associated with either signs of increased disease activity in the wrists, finger joints (although erosions involved these joints), or elbows, or with any generalised flare. Oedema did not involve the legs at any 
time, and no changes in anti-inflammatory drug therapy had been made which could have been related to the episodes of forearm swelling.

PATIENTS WITH UPPER LIMB OEDEMA IN WHOM LYMPHATIC CHANNELS COULD NOT BE DEMONSTRATED

Table 1 sets out the salient clinical features involving these 3 patients, who are cases 3,4 , and 5 . All patients had erosive disease which involved various small joints of the fingers and the wrists.

Case 3 had swelling of the right hand and forearm for about 18 months before the left was similarly affected. This patient's disease was atypical in that massive synovial hypertrophy of the right subacromial bursa of the type described by Kent et al. (1978) was an early sign, and synovectomy of this bursa preceded swelling of the right hand by 9 months. The left subacromial bursa became similarly involved. Swelling of the left hand followed involvement of the ipsilateral shoulder by 18 months, but preceded the synovectomy eventually performed on this side. Case 4 had had oedema of the right hand and wrist for 5 months before lymphangiography was undertaken. This patient also has von Willebrand's disease. Case 5 has chronic progressive disease.

These 3 patients were all receiving a salicylate. In addition case 3 was receiving indomethacin and case 5 ibuprofen. In no case had hypoproteinaemia been detected.

\section{PATIENTS WITH LOWER LIMB OEDEMA}

Table 2 sets out the salient clinical features. Both patients were thought to have developed more extensive oedema of the lower limb than expected from the disease activity of the lower limb joints; nevertheless case -6 had hips, knees, and ankles involved, and case 7 had effusions in both knees. No other cause for the oedema was detected. Both patients were receiving indomethacin and in addition case 6 ibuprofen and case 7 a salicylate.

\section{LYM PHANGIOGRA PHY}

The technique adopted for lymphangiography was that described by Kinmonth (1952). Patent blue dye was injected into all the interdigital webs of the

Table 1 Clinical features of cases 3, 4, and 5

\begin{tabular}{lllrll}
\hline Case & Age & Upper limb & Duration* & SCAT & Erosions \\
\hline 3 & 32 & Bilateral & 3 years & $-\mathrm{ve}$ & ++ \\
4 & 51 & Right & 9 years & $+\mathrm{ve}$ & ++ \\
5 & 51 & Left & 13 years & $+\mathrm{ve}$ & ++ \\
\hline
\end{tabular}

*Disease duration before the appearance of oedema. ${ }^{* *}$ Including the wrists and small joints of the hands.
Table 2 Clinical features of cases 6 and 7

\begin{tabular}{llllll}
\hline Case & Age & Lower limb & Duration* & SCAT & Erosions \\
\hline 6 & 54 & Bilateral & 5 years & +ve & Present \\
7 & 60 & Bilateral & 2 months & - ve & Absent \\
\hline
\end{tabular}

*Disease duration before the appearance of oedema.

affected hand or foot. It was not possible to see subcutaneous lymphatics through the skin in any patient because of the presence of oedema. An exploratory incision was made on the dorsum of the hand or foot just distal to the wrist or ankle joint. Where visible, a subcutaneous lymphatic channel was cannulated and $2 \mathrm{ml}$ of iodised oil ultrafluid (Lipiodol) slowly injected over a period of 40 minutes. Radiographs were obtained immediately after the procedure in case 1 , and in case 2 the procedure was modified and radiographs were obtained at 5-minute intervals during the injection period. In both cases further films were obtained 24 hours later. A similar procedure was followed in the 2 patients in whom the leg lymphatics were studied.

\section{Results}

Radiological evidence of oedema, that is, blurring of the fascial-fat interface, increased depth of the

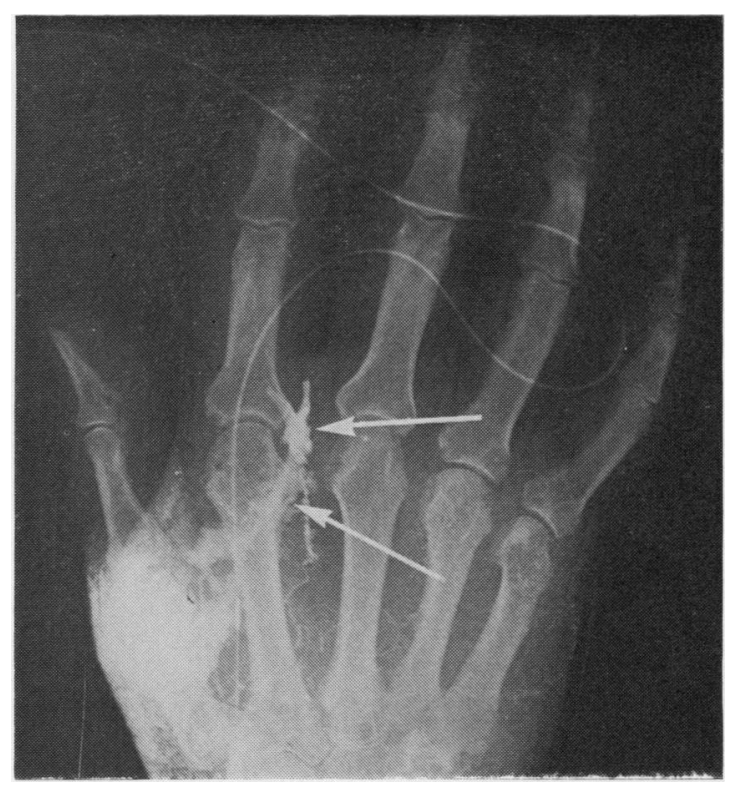

Fig. 1 Case 1. Right upper limb. The catheter tip can be seen proximally in the first intermetacarpal space. Note the dermal back flow and perivenous extravasation of contrast material (arrows). (The contrast material overlying the first metacarpal is due to direct extravasation into the tissues.) 
subcutaneous tissues, and prominence of the neurovascular pedicles, extended more proximally than was judged to be the case clinically.

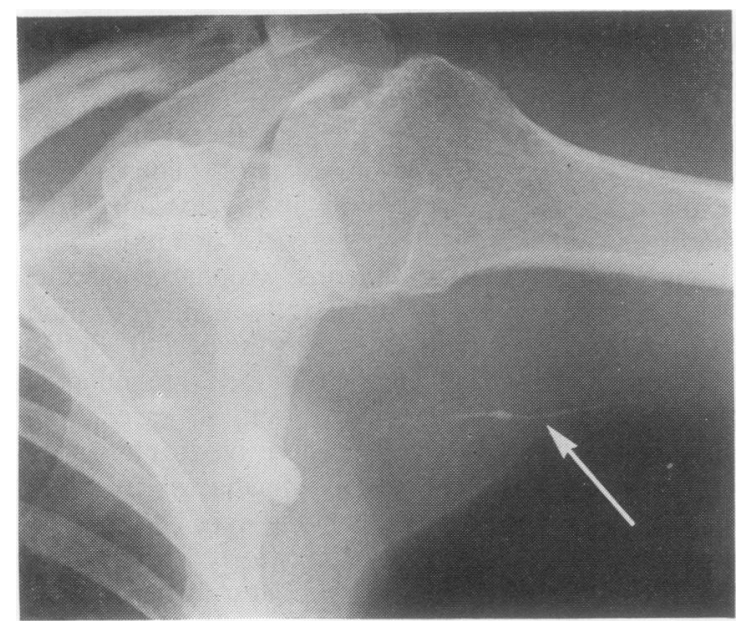

Fig. 2 Case 1. Persistent opacification of a subcutaneous lymphatic 24 h after lymphangiagraphy (arrow). Two axillary lymph nodes are also outlined.

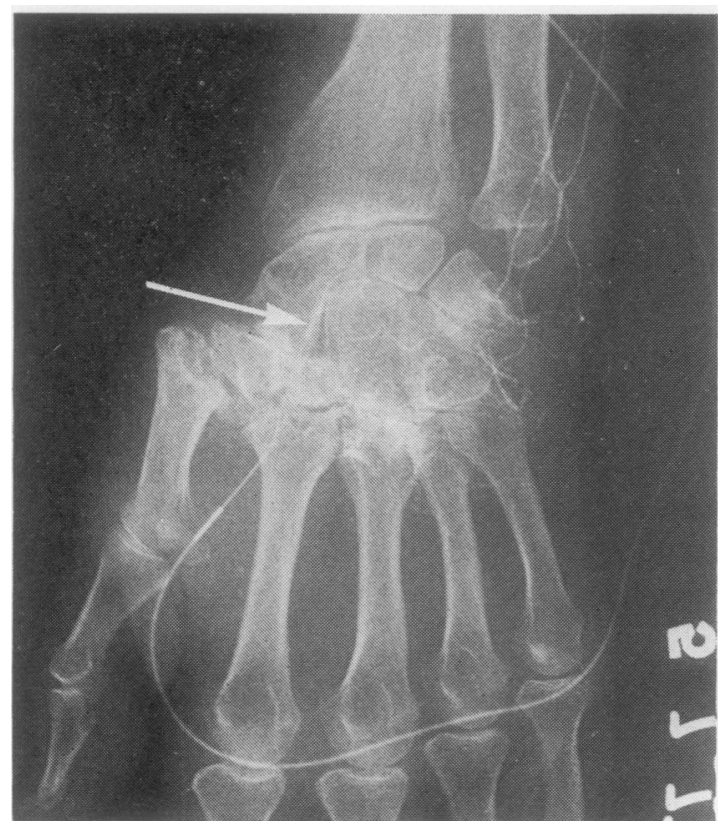

Fig. 3 Case 2. Left upper limb. The cannulated subcutaneous lymphatic can be followed as far as the distal row of carpal bones (arrow).
CASE 1

Complete obstruction of the subcutaneous lymphatic channels associated with dermal backflow into the hand and extravasation into the perivenous tissues was demonstrated after perfusion with Lipiodol (Fig. 1). The dermal backflow obscured the cannulated channel and indicated the need for the serial films taken in the second case. Subcutaneous lymphatic channels of the upper arm were not outlined during the initial examination but were apparent after a period of 24 hours (Fig. 2).

\section{CASE 2}

The cannulated subcutaneous lymphatic channel was outlined as far as the proximal row of carpal bones (Figs. 3 and 4). The contrast medium instead of traversing the subcutaneous lymphatic channel refluxed back into the dermal lymphatics as a result of the obstruction. Lymphatic flow beyond the wrist

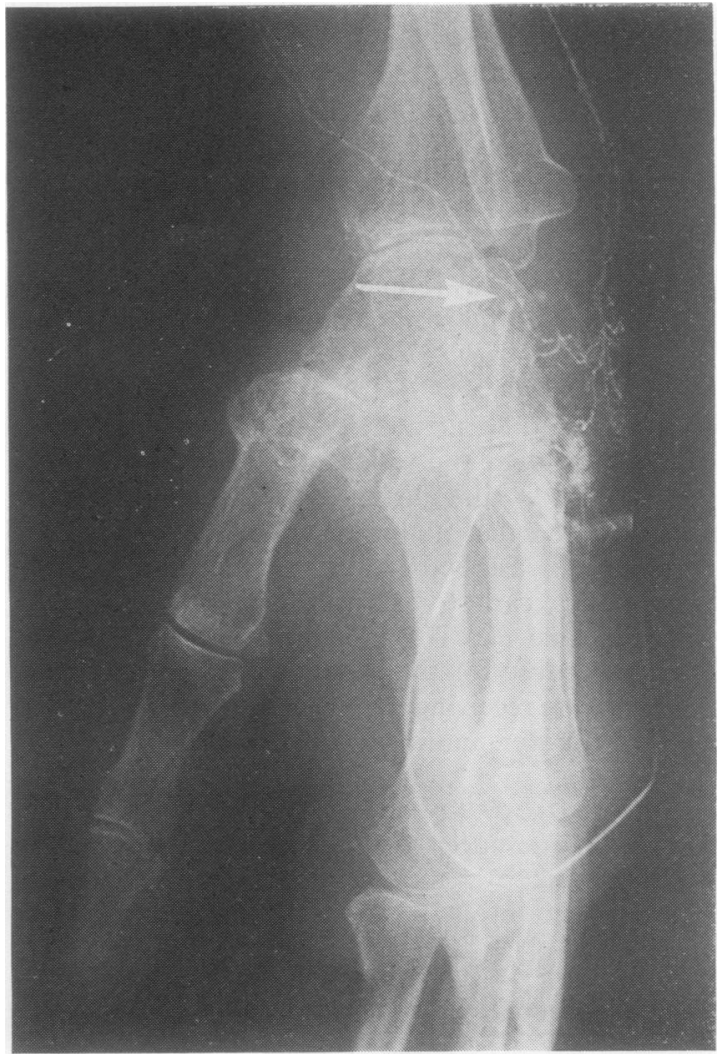

Fig. 4 Case 2. Lateral projection of Fig. 3. Note that reflux into the dermal lymphatics dorsal to the carpus has enabled contrast to reach indirectly the subcutaneous lymphatics of the forearm.

The obstruction is indicated by an arrow. 


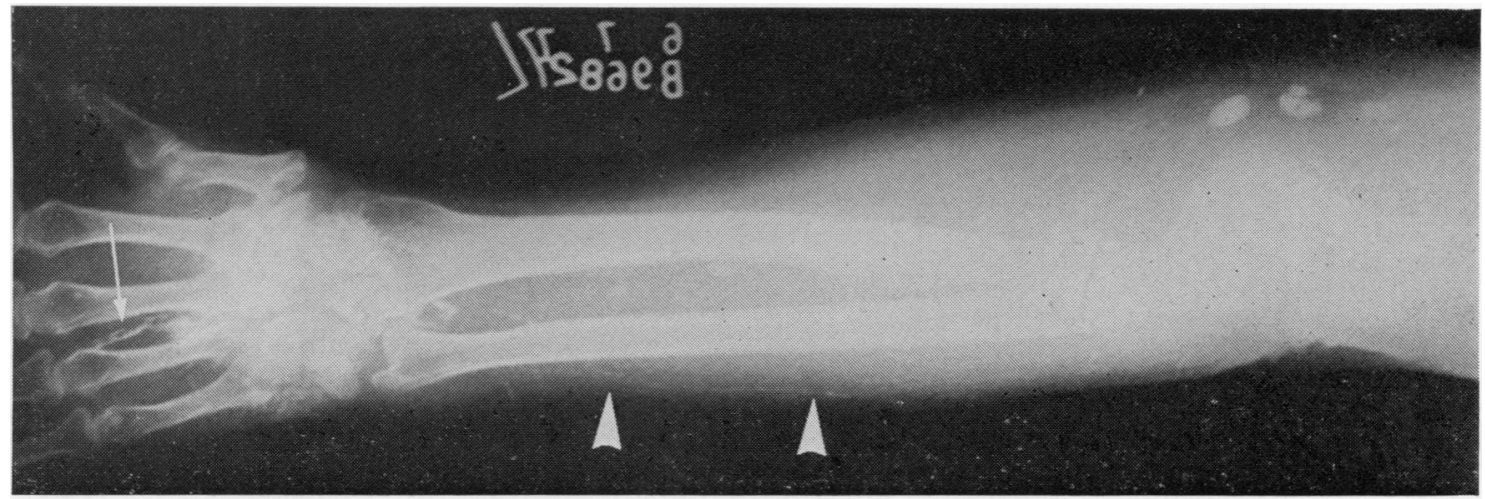

Fig. 5 Case 2. Persistent opacification of forearm lymphatics 24 hours after lymphangiography (arrow heads). As in Case 1, the lymphatic glands (olecranon) show filling defects which reflect nodular hyperplasia. The small arrow indicates perivenous extravasation

joint was maintained only by the dermal lymphatics. The 24-hour films demonstrate perivenous extravasation and lymphstasis (Fig. 5) and lymph nodes containing small filling defects situated in the forearm.

\section{CASES 3,4 , AND 5}

These were the 3 further patients with upper limb oedema in whom no lymphatic channels were found on cut-down following the injection of patent blue dye, and the lymphangiographic procedure was abandoned.

\section{CASES 6 AND 7}

In both of these 2 patients with lower leg oedema normal lymphatic channels were demonstrated. The regional lymph nodes (inguinal, pelvic, and paraaortic) were rounded but showed the accentuated punctate pattern of nodular hyperplasia.

\section{Discussion}

This study has provided convincing lymphangiographic evidence that lymphatic channel obstruction is responsible for diffuse oedema of the hand and forearm in some patients with rheumatoid disease. In both patients with upper limb oedema in whom a subcutaneous lymphatic channel was successfully cannulated the classical changes secondary to mechanical obstruction of the subcutaneous lymphatics-dermal backflow, lymph stasis, and perivenous extravasation-were demonstrated. Physical properties of Lipiodol ultrafluid are such that the lymphatic channels are not outlined unless there has been successful cannulation of a subcutaneous lymphatic. If Lipiodol ultrafluid is injected into the tissues after an unsuccessful cannulation, the con- trast medium remains at the site of injection and does not enter the lymphatics. The phenomenon of dermal backflow indicates that there is obstruction to the onward flow of lymph (Craig, 1969). In a normal subject injection of this contrast medium into a subcutaneous lymphatic results in this channel and its branches being outlined during the onward flow of the medium (Fig. 6). Lymph stasis is reflected in the contrast medium being retained within the lymphatics. Lipiodol is not retained for more than 24 hours within normal lymphatics, and retention beyond this period is in keeping with proximal obstruction. Contrast medium that extravasates from obstructed lymphatics accumulates in the loose perivenous areolar tissues.

In the second patient the contrast medium instead of directly traversing the cannulated subcutaneous lymphatic refluxed back into the dermal lymphatics at the level of the wrist and in this manner bypassed the obstruction. It should be noted that the dermal lymphatics themselves are so minute that they cannot be cannulated, whereas the subcutaneous lymphatics in the hand measure approximately a third of a millimetre in diameter in the undistended state.

In the presence of gross oedema subcutaneous injection of patent blue dye may not outline the lymphatics, as the dye diffuses into the oedematous tissues, and attempts to locate lymphatics may thus be unsuccessful. In the 3 further patients with rheumatoid disease with similar oedema of the forearms it was not possible to demonstrate lymphatics with the patent blue dye technique after cutdown. It is impossible to state whether this observation reflected extensive obliteration of lymphatics in these patients or was the effect of the oedema. Exploratory incision proximal to the edge of the 


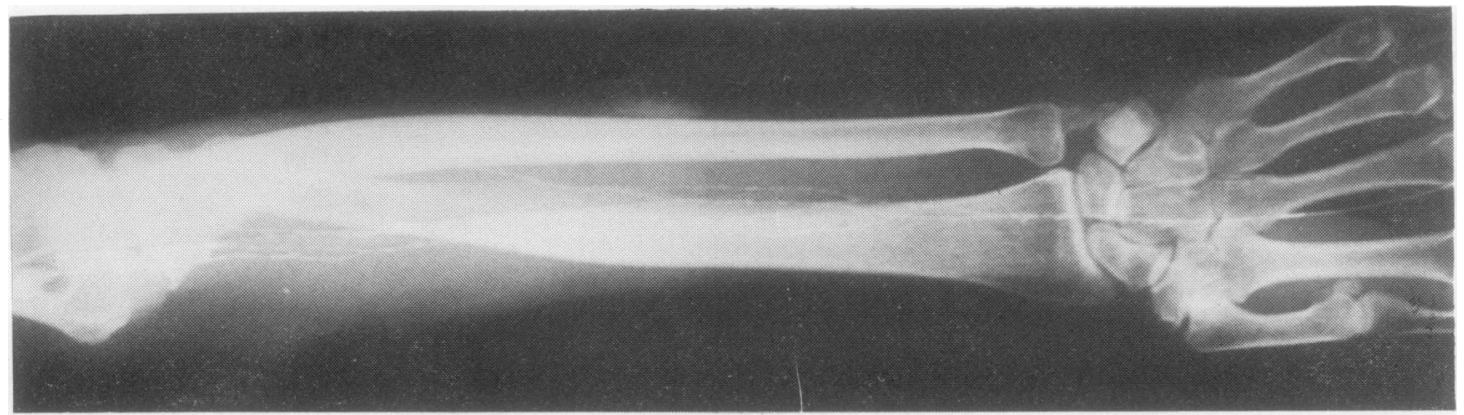

Fig. 6 Normal lymphatic architecture of the upper limb after cannulation and instillation of contrast into a subcutaneous lymphatic channel at the wrist. Note the absence of dermal backflow and other signs of lymph stasis

blue dye as was undertaken in these patients is always worthwhile before a decision is made to abandon this investigation.

In neither patient did the lymph nodes of the upper limb appear to be significantly enlarged either on palpation or on the lymphangiogram, nor was there any evidence to suggest that any obstruction to lymph flow was occurring at the nodes. The filling defects noted were compatible with germinal centre hypertrophy and have been noted previously (Robertson et al., 1968).

All 5 patients with upper limb oedema had erosive rheumatoid disease which involved the wrists, and both patients in whom it was possible to demonstrate lymphatic obstruction were seronegative. In 3 of these 5 patients the oedema became bilateral, and in 1 of the remaining 2 the swelling was of relatively recent onset. Spontaneous regression and temporary regression following distant corticosteroid injection was observed. Pain and signs of inflammation other than that directly associated with adjacent joint disease were not features.

The demonstration of normal lymphatic channels in the 2 patients with lower limb oedema emphasises the difficulty in differentiating the various possible causes of dependent oedema in rheumatoid disease and does not exclude the possibility that similar lymphatic obstruction to that seen in the upper limbs may on occasion involve the legs.

It is possible that an inflammatory arthritis developing in a patient with some pre-existing anatomical or functional deficiency of the lymphatic system might result in peripheral oedema. It is more likely that lymphatic obstruction might result from the direct extension of the inflammatory process from involved joints to the surrounding tissues or be a consequence of immune complexes reaching these channels and initiating a chronic lymphangitis. There was no suggestion, however, that there had been any acute additional event likely to lead to extension of the inflammatory process such as secondary joint infection or synovial rupture.

Our findings are in keeping with the lymphoscintigraphic study of Kalliomaki and Vastamaki (1968), in which evidence of lymphatic obstruction was found in 2 rheumatoid patients with unilateral upper limb oedema. Most previous studies have, however, investigated rheumatoid patients with lower limb oedema (Swinburne, 1964; Jayson and Barks, 1971) and have concluded that lymphatic obstruction was not the cause of oedema. In a subsequent study Jayson et al. (1971) made use of the local clearance rate of intradermally injected radiolabelled albumin as an index of lymphatic flow rates, and, although they found some slowing of clearance rates in their rheumatoid group overall as compared to their controls, they found that this slowing was actually less marked in patients with oedema than in those without and concluded that lymphatic obstruction was not responsible. This study can be criticised on the grounds that locally injected albumin has been shown to be cleared by both venous and lymphatic routes (Sage et al., 1964), suggesting that this method does not give a specific indication of lymphatic flow rate.

The present study has demonstrated that in some patients with rheumatoid oedema of the upper limbs lymphatic obstruction does occur. The clearance rate of the inert gas radioactive xenon $\left({ }^{133} \mathrm{Xe}\right)$ from a region following local injection has been used as an index of the adequacy of its nutrient blood flow and venous drainage (Sejrsen, 1967; Grennan et al., 1975). Normal local clearance rates of xenon were obtained from one of our patients (not performed in the other) in whom lymphangiography demonstrated lymphatic blockage, suggesting that the venous flowwas not significantly impaired in this patient.

One might also speculate whether lymphatic obstruction may contribute to persistent joint 
effusion in rheumatoid disease. Kuhns (1933) described the apparent obliteration of synovial lymphatics in patients with joint inflammation, an observation which suggests that an obliterative lymphangitis may accompany synovitis. Weston (personal communication), however, regards contrast filled lymphatics as a rather specific feature which accompanies the rheumatoid arthrogram. These apparently opposing observations are difficult to reconcile unless the latter phenomenon represent dilatation of residual channels.

Financial assistance was provided by the Medical Research Council of New Zealand.

\section{References}

Craig, J. O. M. C. (1969). Lymphangiography. In Textbook of Radiology. Edited by D. Sutton and R. G. Granger. 1st edn., pp. 607-617. E. \& S. Livingstone: London.

Grennan, D. M., Rooney, P. J., St. Onge, R. A., Brooks, P. M. Zeitlin, I. M., and Dick, W. C. (1975). Histamine receptors in the synovial microcirculation. European Journal of Clinical Investigation, 5, 75.

Jayson, M. I. V., and Barks, J. S. (1971). Oedema in rheu- matoid arthritis. Changes in the coefficient of capillary filtration. British Medical Journal, 2, 555-557.

Jayson, M. I. V., Cavill, I., and Barks, J. S. (1971). Lymphatic clearance rates in rheumatoid arthritis. Annals of the Rheumatic Diseases, 30, 638-639.

Kalliomaki, J. L., and Vastamaki, M. (1968). Chronic diffuse oedema of the rheumatoid hand. A sign of local lymphatic involvement. Annals of the Rheumatic Diseases, 27, 167-169.

Kent, A. H., Nelson, A. M., and Hunder, G. G. (1978). Shoulder swelling in rheumatoid arthritis secondary to subacromial bursitis. Arthritis and Rheumatism, 21, 145147.

Kinmonth, J. B. (1952). Lymphography in man. Clinical Science, 11, 13-20.

Kuhns, J. G. (1933). Lymphatic drainage of joints. Archives of Surgery, 27, 345-391.

Robertson, M. D. J., Hart, F. D., White, W. F., Nuki, G., and Boardman, F. L. (1968). Rheumatoid lymphadenopathy. Annals of the Rheumatic Diseases, 27, 253-260.

Sage, H. H., Sinha, B. K., Kizilay, D., and Toulon, R. (1964). Radioactive colloidal gold measurements in lymph flow and functional patterns of lymphatics and lymph nodes in the extremities. Journal of Nuclear Medicine, 5, 626-642.

Sejrsen, P. (1967). Cutaneous blood flow in man studied by freely diffusible radioactive indicators. Scandinavian Journal of Clinical and Laboratory Investigation, Suppl. 93, 52-59.

Swinburne, K. (1964). Oedema of feet and ankles in rheumatoid arthritis. British Medical Journal, 1, 1541-1544. 